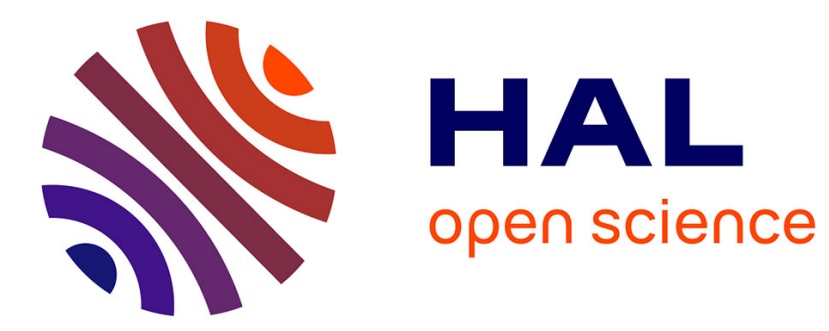

\title{
Le présentiel allégé à l'université pour les grands groupes. Un dispositif au service de l'autonomisation des apprenants
}

Thierry Soubrié

\section{- To cite this version:}

Thierry Soubrié. Le présentiel allégé à l'université pour les grands groupes. Un dispositif au service de l'autonomisation des apprenants. Distances et savoirs, 2007, 5 (1), pp.13-28. 10.3166/ds.5.13-28 . hal-03409626

\section{HAL Id: hal-03409626 \\ https://hal.science/hal-03409626}

Submitted on 29 Oct 2021

HAL is a multi-disciplinary open access archive for the deposit and dissemination of scientific research documents, whether they are published or not. The documents may come from teaching and research institutions in France or abroad, or from public or private research centers.
L'archive ouverte pluridisciplinaire HAL, est destinée au dépôt et à la diffusion de documents scientifiques de niveau recherche, publiés ou non, émanant des établissements d'enseignement et de recherche français ou étrangers, des laboratoires publics ou privés. 


\title{
LE PRÉSENTIEL ALLÉGÉ À L'UNIVERSITÉ POUR LES GRANDS GROUPES
}

Un dispositif au service de l'autonomisation des apprenants

Thierry Soubrié

\section{Lavoisier | Distances et savoirs}

\author{
2007/1 - Vol. 5 \\ pages 13 à 28
}

ISSN 1765-0887

Article disponible en ligne à l'adresse:

http://www.cairn.info/revue-distances-et-savoirs-2007-1-page-13.htm

Pour citer cet article :

Soubrié Thierry, « Le présentiel allégé à l'université pour les grands groupes » Un dispositif au service de l'autonomisation des apprenants,

Distances et savoirs, 2007/1 Vol. 5, p. 13-28. DOI : 10.3166/ds.5.13-28

Distribution électronique Cairn.info pour Lavoisier.

(c) Lavoisier. Tous droits réservés pour tous pays.

La reproduction ou représentation de cet article, notamment par photocopie, n'est autorisée que dans les limites des conditions générales d'utilisation du site ou, le cas échéant, des conditions générales de la licence souscrite par votre établissement. Toute autre reproduction ou représentation, en tout ou partie, sous quelque forme et de quelque manière que ce soit, est interdite sauf accord préalable et écrit de l'éditeur, en dehors des cas prévus par la législation en vigueur en France. II est précisé que son stockage dans une base de données est également interdit. 


\title{
Le présentiel allégé à l'université pour les grands groupes
}

\section{Un dispositif au service de l'autonomisation des apprenants}

\section{Thierry Soubrié}

\author{
LIDILEM Université Stendhal, 1180 avenue Centrale - Domaine Universitaire \\ F-38400 Saint Martin d'Hères, BP 25, F-38040 Grenoble cedex 9 \\ Thierry.Soubrie@u-grenoble3.fr
}

\begin{abstract}
RÉSUMÉ. Cette communication propose de rendre compte d'une expérience d'hybridation d'un cours de première année de master de sciences du langage à l'université Stendhal de Grenoble 3 intitulé "technologies de l'information et de la communication pour l'enseignement/apprentissage du français langue étrangère ». Le choix d'un dispositif hybride s'est imposé suite à la décision de rendre ce cours obligatoire. De plus en plus de formations en langues intègrent en effet l'utilisation des TICE, et il devenait important de sensibiliser l'ensemble des étudiants à cette question. Il restait à résoudre le problème de l'effectif, qui a triplé après que le cours est devenu obligatoire, et à conserver la qualité de l'encadrement qui était offerte jusqu'alors (alternance de cours théoriques et de TD). Répondre à ce questionnement à amené à prendre en considération une dimension de l'enseignement/apprentissage sous-estimée jusqu'ici : l'autonomisation des apprenants. Après avoir détaillé le dispositif et expliqué les choix faits, cet article dresse le bilan d'une année de fonctionnement et expose en conclusion certains des principes et des contraintes inhérents à ce type de formation.
\end{abstract}

ABSTRACT. This article is an account of experimenting a blended learning course applied to a course of master of linguistics (first year) entitled "technologies for teaching/learning of French as a foreign language" at Stendhal university (Grenoble 3). Choosing blended learning is a consequence of this course becoming compulsory. More and more language courses integrate educational technology, and it is important that all students should be aware of this. There remained the problem of facing the number of students that trebled, resulting from the course being compulsory, while ensuring equal quality. Solving this lead to take into consideration a dimension of teaching/learning underestimated up to then: making learners autonomous and self-directed. After describing the system and explaining the reasons for the choices, the present paper draws up an assessment of this one year experiment and concludes with some of the principles and constraints inherent to this type of teaching.

MOTS-CLÉS: formation hybride, autonomisation, TICE, dispositif, pédagogies actives, apprentissages collaboratifs.

KEYWORDS: blended learning, self-directed learning, e-learning, device, learning by doing, collaborative learning. 


\section{Introduction}

C'est en 1999 que le cours intitulé «technologies de l'information et de la communication pour l'enseignement du français langue étrangère » a fait son entrée dans la maquette de la maîtrise FLE de l'université Stendhal, diplôme très nettement orienté vers la professionnalisation des étudiants. C'était à cette époque un enseignement optionnel de $50 \mathrm{~h}$ qui s'étalait sur les deux semestres de l'année universitaire. Choisi par une petite vingtaine d'étudiants, il pouvait se dérouler dans une salle informatique, permettant ainsi à l'enseignant d'alterner à loisir les phases de cours magistral et de TD. A l'occasion du passage au LMD, j'ai obtenu de la part de l'UFR des sciences du langage et de l'université que ce cours devienne obligatoire. De plus en plus de formations en langues intègrent en effet l'utilisation des TICE, que ce soit à travers la mise en place de centres de ressources ou le développement de formations ouvertes et à distance, et il devenait important de sensibiliser l'ensemble des étudiants à cette question. Il restait à résoudre le problème de l'effectif, puisque le cours, en devenant obligatoire, a vu le nombre d'étudiants passer de 20 à 60. Comment faire face à une telle augmentation sans pour autant perdre la qualité de l'encadrement qui était offerte jusqu'ici ? C'est à partir de ce questionnement et après avoir étudiés différents types de scénarios (cf. typologie Competice ${ }^{1}$ ) que $\mathrm{j}$ 'ai été amené à prendre en considération une dimension de l'enseignement/apprentissage que j'avais sous-estimée jusqu'ici : l'autonomisation des apprenants. Je me propose dans cette communication de détailler le dispositif vers lequel je me suis tourné, d'expliquer les raisons de mes choix et de faire le bilan d'une année de fonctionnement.

\section{Cadre théorique}

Comme cela se passe pour tout nouveau support d'information, les TIC remettent en cause les circuits de diffusion et de légitimation de l'information. C'est à l'utilisateur désormais, placé au premier plan, de se confronter directement aux informations, de les sélectionner, de les réorganiser et de se les approprier (Ollivier, 2000). Or, compte tenu de la complexité de ces supports de l'écrit, qui substituent à l'organisation traditionnellement hiérarchique des contenus, un système basé sur le principe des associations libres, la tâche n'est pas aisée. Contrairement à ce que pourrait laisser croire le mythe persistant de l'immatérialité du numérique (Jeanneret, 2002), les opérations de lecture/écriture sur internet sont d'une grande complexité. L'utilisateur en effet doit mener de front différentes tâches : repérer les liens hypertextuels, comprendre la sémiotique complexe dont ils relèvent (leur place dans le document, leur autoréférentialité et leur fonction instrumentale), identifier les différentes zones que comporte une même page (menus déroulants, zones de saisie, zones de validation, frames, etc.), tenir compte des modifications que 
subissent les documents dans le temps (mises à jour), jongler avec différents types de stratégies d'exploitation de l'interface (mono vs. multifenêtrage, affichage plein écran vs. affichage des fenêtres en taille réduite), gérer les réactions et les interventions parfois inopportunes du système (ouverture de fenêtres pop-up par exemple), etc. Sans compter la difficulté d'appréhender la profondeur du web à travers l'espace exigu de l'écran (Jeanneret, 2000 ; Souchier 2003).

En d'autres termes, les TIC réaffirment l'importance de l'autonomie de l'utilisateur. C'est même selon Monique Linard une condition de leur efficacité. Mais cela n'est pas sans conséquences sur «le monde du travail et de la connaissance et par suite, de la formation d'adulte et de l'éducation » (Linard, 2002). Car la formation à l'autonomie n'est pas compatible avec le modèle encore très largement répandu dans les systèmes éducatifs, qui repose sur l'hétéronomie et la transmission des contenus. On sait en effet depuis les premiers travaux du CRAPEL sur le sujet, que l'autonomie n'est pas innée chez l'individu (Holec, 1990). Il importe de mettre en place des formations spécifiques qui alternent des temps de travail en autonomie et des temps d'accompagnement où l'accent est avant tout mis sur le savoir apprendre. L'action pédagogique part des besoins exprimés par l'apprenant et du constat qu'il fait en relation avec l'enseignant de ses connaissances et compétences. Dans cette démarche, la dimension métacognitive est centrale. Le développement de la capacité à apprendre passe par l'évolution des représentations des apprenants quant à leurs conceptions de la matière enseignée et des méthodes d'apprentissage. Le rôle du formateur consiste à mettre en place les conditions favorables à ce retour réflexif, notamment en construisant des activités ancrées dans le réel et qui ont du sens pour les apprenants.

La notion de dispositif joue dans ce cadre un rôle important. Habituellement employé dans un contexte technique - il désigne alors un ensemble de pièces constituant un mécanisme -, il est utilisé en ingénierie de la formation pour désigner un ensemble de moyens matériels et humains, agencés en vue de faciliter un processus d'apprentissage (Lameul, 2001). Cette évolution n'est pas anodine. Tout d'abord, elle donne aux objets, les ordinateurs en particulier, le statut d'outils cognitifs, capables d'influer notablement sur l'activité du sujet (on ne lit pas par exemple de la même façon un texte sur support imprimé ou sur support numérique (Soubrié, 2005)). L'intelligence n'est plus considérée comme appartenant en propre aux individus, mais partagée avec les outils, les artefacts, présents dans l'environnement (Salembier, 1996). L'autre conséquence de cette évolution est que l'enseignement, autrefois perçu, disons jusqu'à l'émergence de la notion de « formation ouverte » dans le champ des sciences de l'éducation, c'est-à-dire au début des années 90, comme un processus essentiellement linéaire et prévisible, est désormais conçu comme un ensemble complexe d'interactions entre les différentes composantes d'une situation d'enseignement/apprentissage. Dès lors, il s'agit moins de planifier un enchaînement d'actions que de prévoir des scénarios d'utilisation :

[...] concevoir un système de formation revient moins à prévoir un chemin idéal qu'à cartographier et à baliser des parcours d'actions possibles. Plutôt 
que d'imposer sa voie à l'apprenant, on met à sa disposition tous les moyens cognitifs, techniques et humains, nécessaires à sa propre conduite, y compris la compétence des enseignants et la collaboration avec les pairs » (Linard, 2002).

On voit bien à travers cette définition qu'il n'est plus question de dispositifs fermés, à l'instar de CD-Rom, mais d'environnements ouverts qui ne limitent plus la communication aux interactions homme-machine, mais incluent également les interactions entre pairs ou avec l'enseignant. Cette évolution témoigne de l'importance qu'ont prises depuis le milieu des années 90 les théories socioconstructivistes et interactionnistes dans l'apprentissage assisté par ordinateur. Le modèle de construction de l'intelligence élaboré en psychologie cognitive reste d'actualité mais il est redéfini dans le cadre de l'approche socioculturelle et de nouvelles théories comme la cognition située, partagée ou distribuée qui insistent sur le rôle que jouent les environnements technique et humain dans la (co)construction des connaissances (Legros et al., 2002).

Cette dimension sociale de l'apprentissage fait partie intégrante de toute démarche basée sur l'autonomisation des apprenants. Contrairement à l'idée qui a longtemps prévalu dans le domaine de l'apprentissage des langues assisté par ordinateur (ALAO), l'autonomie ne signifie pas travailler de manière isolée. $\mathrm{Ne}$ serait-ce que parce que le travail en groupe, notamment la collaboration, c'est-à-dire lorsque chacun des membres du groupe doit réaliser le même travail que les autres avant de se mettre d'accord sur une version commune, requiert que chaque participant soit autonome (Mangenot, 2003). Mais aussi parce qu'être autonome, c'est aller chercher l'information où elle se trouve, et pas seulement dans les tutoriels, mais auprès de personnes ressources. C'est pourquoi dans la liste des grands domaines d'application de l'autonomie que dresse Brigitte Albero, les compétences «sociales» tiennent une place importante : «communiquer pour apprendre », «faire des situations d'échange des occasions d'apprentissage », " coopérer, échanger, partager l'information", "se construire un réseau de personnes ressources », " demander et obtenir de l'aide », " développer une attitude d'ouverture, de tolérance, d'empathie avec ses interlocuteurs » (Albero, 2003). Se construire comme un sujet autonome nécessite que l'on se pense comme sujet d'une collectivité, un sujet dont la « vérité propre » est toujours «participation à une vérité qui le dépasse, qui s'enracine finalement dans la société et dans l'histoire » (Albero, 2002).

\section{Modèle pédagogique}

Le cours dont j'ai la charge est une introduction à la problématique de l'intégration des TIC dans l'enseignement/apprentissage des langues et du français langue étrangère en particulier. Il s'agit de faire prendre conscience aux étudiants que, fondamentalement, ce que les TICE remettent en cause, c'est le statut et les 
fonctions de chacun, l'enseignant étant appelé à jouer un rôle de tuteur, d'accompagnateur, et les apprenants à prendre une part de responsabilité accrue dans leur apprentissage. Sensibiliser de futurs enseignants, inscrits pour la plupart d'entre eux en formation initiale, à cette nouvelle répartition des tâches est relativement délicat puisque la prise de conscience doit intervenir à deux niveaux. En tant qu'apprenant, le cours doit les conduire, à travers les mises aux points théoriques et le dispositif mis en place, à devenir plus autonomes. En qualité de futurs enseignants, ils doivent être en mesure d'élaborer des scénarios pédagogiques qui prennent en compte ces changements.

Pour mener à bien ce programme, j'ai choisi d'adopter une démarche tantôt explicite, lorsque je m'adressais à eux comme à de futurs enseignants et qu'il s'agissait de faire le point sur des questions comme l'autoapprentissage ou la formation ouverte et à distance, tantôt implicite, dès lors qu'il était question d'amener les étudiants à faire évoluer leurs représentations sur leurs propres pratiques. Dans un cas, j'avais recours à des cours magistraux et à des activités de mise en pratique (comparaison de sites didactiques d'inspiration différente pas exemple), dans l'autre, à un dispositif autonomisant, suivant en cela un des préceptes fondateurs des pédagogies actives formulé par John Dewey : «Learning by doing ». C'est l'alternance entre ces deux démarches qui devait permettre aux étudiants de comprendre ce que signifie l'autonomie du point de vue de l'enseignant et de l'apprenant.

\section{Description du dispositif}

Dans la perspective de développer l'autonomie intellectuelle des étudiants, j'ai accordé tout autant d'importance aux activités d'analyse (étudier les changements qu'induisent les TIC dans l'histoire de l'évolution des supports de l'écrit et de la lecture, différencier les produits selon qu'ils se situent dans une perspective behavioriste ou constructiviste, s'interroger sur l'évolution des technologies éducatives vers les apprentissages collectifs, etc.) qu'aux activités de conception de matériel didactique (élaboration d'exercices autocorrectifs, de parcours d'apprentissage, de scénarios pédagogiques, etc.). L'analyse critique était également encouragée à travers l'évaluation puisque les tâches proposées aux étudiants comportaient toujours une partie réflexive sous la forme d'une fiche pédagogique dans laquelle ils devaient expliciter et justifier leur démarche.

Mais soutenir l'autonomie, c'est aussi donner la possibilité aux étudiants de choisir un mode de formation plus ou moins guidé. C'est pourquoi, à côté du cours magistral obligatoire de $24 \mathrm{~h}$, étaient proposées des séances facultatives de tutorat informatique, encadrées $4 \mathrm{~h}$ par semaine par un des étudiants de la promotion, et des séances de tutorat pédagogique. Ces dernières étaient assurées par une enseignante experte dans l'enseignement du FLE et par moi-même. Elles étaient l'occasion d'accompagner certains des étudiants dans les activités que je leur proposais d'une 
semaine sur l'autre en leur fournissant à la fois une aide méthodologique, technique et didactique. Enfin, de façon à ne pas pénaliser les étudiants ne disposant pas d'ordinateur personnel ou de connexion à internet, six heures réparties sur la semaine étaient également prévues pour une utilisation libre d'une salle équipée d'une douzaine d'ordinateurs.

L'autre choix que les étudiants avaient à faire concernait les modalités d'évaluation. Quatre tâches leur étaient proposées parmi lesquelles ils devaient en choisir trois : (1) rédaction d'une fiche descriptive et critique d'un site ou d'une partie d'un site d'apprentissage du FLE ou d'un CD-Rom, (2) élaboration d'un parcours d'apprentissage à partir de ressources pédagogiques sur internet et de CDRom d'autoapprentissage du FLE, (3) conception d'un scénario pédagogique axé sur le développement des compétences de lecture, (4) création d'une tâche ou d'un scénario pédagogique à partir de sites internet non destinés à l'apprentissage du français. En outre, de manière à encourager les étudiants à s'impliquer de manière continue dans le cours et à travailler en dehors du strict cadre universitaire, ils pouvaient être dispensés d'une des tâches s'ils participaient régulièrement aux activités que je leur proposais d'un cours sur l'autre sur une plateforme de travail collectif.

C'est la plateforme Dokeos, anciennement baptisée Claroline, que nous avons utilisée. Proposée par Greco (GREnoble universités Campus Ouvert), structure interuniversitaire chargée depuis 1999 de conduire une stratégie commune de déploiement des TICE, elle dispose, outre des fonctionnalités habituelles que l'on retrouve sur ce type de produit (agenda, liste des membres, forums, partage de fichiers, mise en ligne de documents, etc.) d'un outil qui permet de créer des séquences d'apprentissage, découpées en modules puis en étapes. Comme cela est souligné dans le manuel du concepteur de cours, les utilisations peuvent être variées. La séquence peut être organisée « en fonction d'un contenu, il constituera alors une sorte de table des matières, ou bien en fonction d'activités, il s'apparentera alors à un agenda de « choses à faire » pour acquérir la maîtrise d'un savoir, d'une compétence ». Je m'en suis servi quant à moi comme d'un échéancier. Un module correspondait à un chapitre du cours et chaque étape à une ou des activités à faire d'une semaine sur l'autre (cf. copie-écran). C'était la condition pour que les étudiants réalisent les activités au rythme imposé par le déroulement du cours en présentiel.

Une place de choix a été réservée au travail collectif. Les réponses aux questions posées sur la plateforme ont ainsi été publiées sur des forums de discussion, dans l'espoir que cela conduise sinon au débat du moins à l'échange d'expériences. De même, les travaux que les étudiants devaient me rendre à la fin du cours ont été réalisés par groupes de deux et mis en ligne sur Dokeos de façon à permettre à chacun d'avoir accès aux productions de tous. Ces mises en commun avaient pour objectif d'encourager le partage des connaissances et la constitution d'une communauté d'apprentissage. 


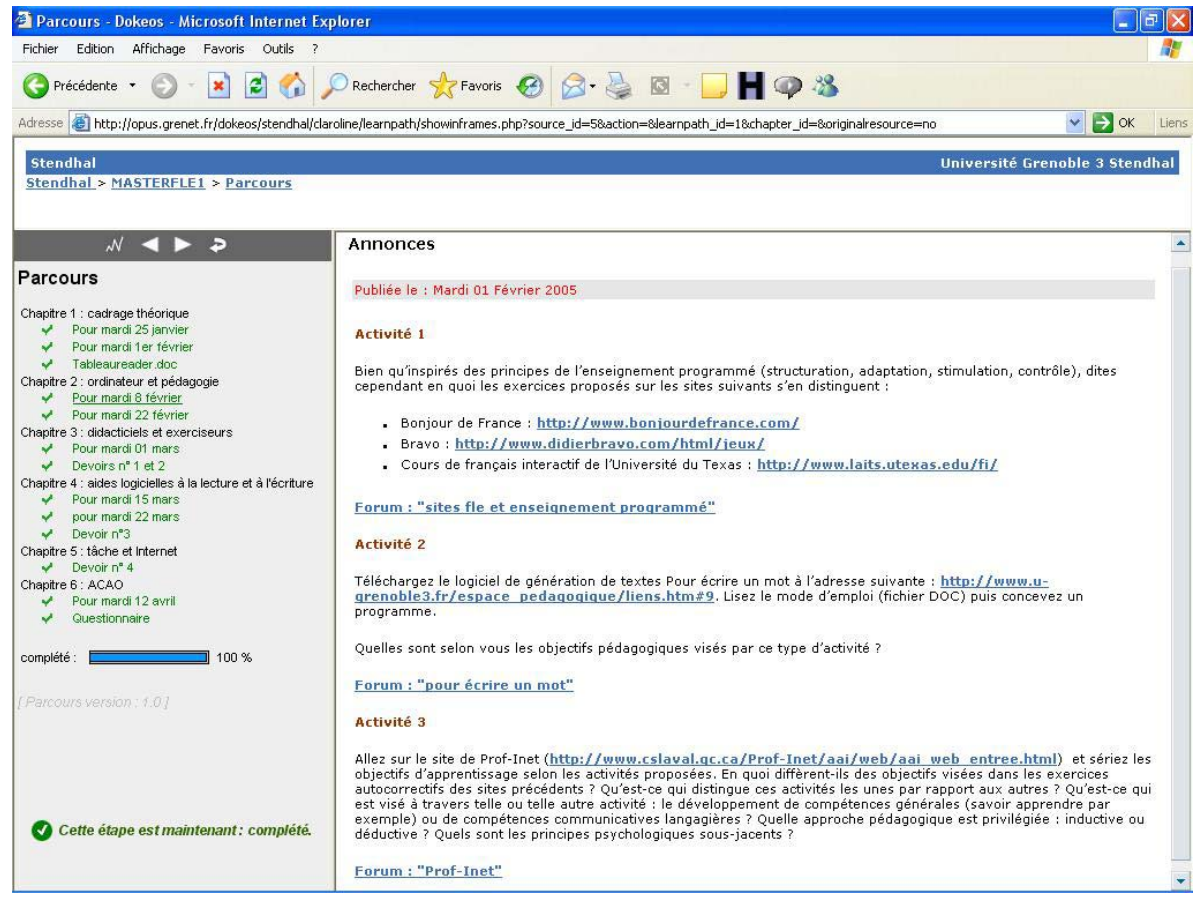

Figure 1. Copie-écran de l'outil parcours de Dokeos ${ }^{2}$

A côté de l'élaboration d'un dossier à deux, une autre activité a nécessité de la part des étudiants qu'ils mettent en œuvre de réelles procédures de collaboration. Il s'agissait de compléter une grille d'analyse critique d'hypermédias d'apprentissage des langues donnée en cours à partir d'autres listes de critères trouvées sur la Toile. Pour ce faire, les étudiants avaient à leur disposition un wiki, site web qui permet l'écriture collaborative, et un forum de discussion pour que chacun puisse commenter et argumenter ses propres modifications ou celles des autres.

Toutes les séances de tutorat se déroulaient en outre par petits groupes de façon à favoriser l'entraide.

Comme du temps de la recherche sur les environnements interactifs d'apprentissage avec ordinateur (EIAO), toute la difficulté a consisté à trouver un

2. Pour chaque chapitre du cours sont proposées des activités et des devoirs ( 4 en tout parmi lesquels il faut en choisir 3). Les 3 activités proposées ici ont été mises en ligne le 2 février 2005. Les étudiants avaient jusqu'au 8 février pour en faire au moins une des trois en répondant aux questions sur les forums (la date est soulignée dans la barre de gauche). Les activités 2 et 3 ont fait l'objet de séances de tutorat pédagogique. 
juste équilibre entre liberté, contraintes et guidage. Dans la définition que donne Henri Portine de l'autonomie, il insiste bien sur cet aspect :

« L'autonomie, c'est construire un projet d'action et gérer la réalisation de ce projet au sein d'une structure qui définit les contraintes globales et apporte une aide lorsqu'elle est nécessaire » (Portine, 1998).

Le dispositif décrit ici se présente en quelque sorte comme une tentative d'instrumentation de l'autonomie. Il fixe un cadre général, qui comporte des mesures obligatoires (les cours magistral, les devoirs), optionnelles (rendre 3 devoirs ou 2 et participer régulièrement aux activités sur la plateforme) et facultatives (tutorat informatique et pédagogique), à l'intérieur duquel les apprenants sont libres d'évoluer, de s'orienter, de faire leurs choix. Reste à savoir si la structure ainsi définie permet réellement aux étudiants d'apprendre autrement, et si les règles qui ont été instaurées n'ont pas été de ce point de vue contreproductives. Monique Linard a plusieurs fois insisté sur le risque d'aboutir à travers la mise en place de dispositifs de ce type, à l'injonction paradoxale suivante : «Je t'ordonne d'être autonome » (Linard, 2003). Jusqu'où peut-on programmer l'autonomisation des apprenants?

\section{Méthodologie}

Pour effectuer mon analyse, j'ai en premier lieu pris en compte les traces laissées par les étudiants sur la plateforme, à savoir les statistiques de connexion et les interactions. Les premières m'ont permis de me faire une idée du taux de participation des étudiants aux différentes tâches proposées et les secondes d'étudier plus finement le contenu des messages et d'étudier en particulier l'impact du modèle pédagogique sur la communication. C'est d'ailleurs pour aller plus loin dans ce sens que je leur ai soumis un questionnaire à la fin du cours afin de me faire une idée plus précise de leur perception du dispositif. 38 questions à choix multiples leur ont été posées, avec la possibilité parfois de donner d'autres réponses que celles proposées (case « autres»). Enfin, je me suis appuyé sur le mémoire de recherche d'une des étudiantes de première année de master FLE avec qui j'ai justement élaboré le questionnaire, et dont le projet était de rendre compte des représentations des étudiants à l'égard des TICE et de leur rôle dans l'enseignement/apprentissage (Sellier, 2005).

\section{Résultats}

\section{Eveil d'une communauté d'apprentissage}

Sur les 79 étudiants inscrits, 62 m’ont rendu les deux ou trois travaux prévus. Un peu plus de la moitié d'entre eux a participé régulièrement aux activités proposées 
sur la plateforme (7 activités sur 9). On enregistre ainsi pour chaque forum de discussion entre 32 et 61 messages.

\begin{tabular}{|l|c|c|}
\hline Discussions & Sujets & Messages \\
\hline Discussion 1 : TICE et mythes & 25 & 37 \\
\hline Discussion 2 : A propos des hyperfictions & 25 & 37 \\
\hline Discussion 3 : EAO et micromondes & 31 & 51 \\
\hline Discussion $4:$ sites web des méthodes FLE & 15 & 35 \\
\hline $\begin{array}{l}\text { Discussion 5 : construction d'une grille commune d'analyse } \\
\text { de CD-ROM }\end{array}$ & 25 & 61 \\
\hline Discussion 6 : à propos de Tropes & 21 & 47 \\
\hline Discussion 7 : aides logicielles à l'écriture & 18 & 32 \\
\hline $\begin{array}{l}\text { Discussion 8 : apprentissages collectifs en ligne et autonomie } \\
\text { de l'apprenant }\end{array}$ & 10 & 24 \\
\hline
\end{tabular}

Tableau 1. Nombre de messages échangés pour chaque discussion

Celui qui a rencontré le plus de succès est sans surprise le forum lié à la tâche collaborative dont il a déjà été question plus haut. Pour se mettre d'accord sur une grille commune d'analyse de didacticiels de langues, certains étudiants sont en effet intervenus plusieurs fois au cours de la semaine. Sur les 28 étudiants qui ont répondu au questionnaire, 23 déclarent avoir lu les messages de leurs camarades avant de publier leurs propres commentaires et 19 en avoir tenu compte lors de la rédaction de leurs messages. Il est intéressant de constater à cet égard combien la prise en compte de cette dimension collective de la communication a été progressive. Au début en effet, on ne note que très rarement des renvois entre les messages, excepté de la part de ceux qui sont déjà très familiers de la communication sur Internet. Lors de la première activité, pour laquelle il fallait réagir à une liste de déclarations quelque peu naïves faites à propos des TIC (Ollivier, 2000), on relève par exemple un texte rédigé par un étudiant amateur de jeux en réseaux qui, pour donner son point de vue, s'appuie sur les propos de ses camarades (Hélène, Iseline, Anne-Marie), alternant approbations (reprise à son compte d'une citation), réfutations (négation, «je ne suis pas d'accord») et concession (modalisée : « peut-être [...] mais ») :

« Les dimensions du monde, à mon humble avis, n'ont pas vraiment été changées. «Le langage est vide » nous dit Hélène, et c'est bien par des phrases comme «les dimensions du monde changent» qu'il perd sa substance. Je ne suis pas d'accord avec Iseline : des lettres ne remplaceront jamais les gens, que les messages arrivent instantanément ou non: la dimension du monde a-t-elle changé grâce au téléphone ? Peut-être dans le cas d'Anne-Marie, qui a pu échanger son appartement et visiter un autre 
pays, cette affirmation commence-t-elle à avoir du sens, mais autrement, Internet n'offre que de pâles alternatives à des modes de communication déjà existants (journaux, livres de voyages, amis étrangers...) »

Cette dimension dialogique est quasiment absente des autres productions, sinon par une référence à l'auteur qui a rapporté ces lieux communs : «Je suis assez d'accord avec Ollivier». L'essentiel des arguments est tiré de l'expérience personnelle des étudiants ou de diverses ressources imprimées ou numériques.

Cela s'explique de plusieurs façons. Le poids des habitudes tout d'abord amène les étudiants à considérer ce type d'activité comme un exercice purement scolaire dont l'objectif est avant tout évaluatif. Dans ce cas, la tendance est de faire preuve d'érudition, de disserter (les premiers messages publiés sont très longs), en faisant la synthèse de discours savants, au lieu de mener une réflexion collective. On peut dès lors se poser la question de l'intérêt que représente la prise en compte de la participation des étudiants sur le forum dans la notation finale. On ne peut pas non plus omettre l'influence d'une certaine tradition scolaire qui condamne le plagiat et promeut l'expression personnelle. La forme des premiers messages s'explique aussi par la mise en page choisie pour le premier forum : la consigne de travail, précisée dans l'outil parcours de la plateforme, est répétée dans le forum. Si bien que les étudiants ont eu tendance à répondre aux questions posées en insérant leur texte sous le texte de la consigne (bouton « répondez sur ce message »), au lieu de créer de nouveaux fils de discussion (bouton : «Lancer un nouveau sujet »). Nul doute que cette façon de procéder, abandonnée par la suite, a renforcé l'idée chez les étudiants que l'activité avait pour seul but de contrôler leurs connaissances.

Quoi qu'il en soit, la façon d'intervenir des étudiants sur les forums a évolué, ceux-ci prenant l'habitude avec le temps de se positionner les uns par rapport aux autres. Cela n'a jamais conduit à l'élaboration de documents finalisés, mais progressivement, les discussions n'ont plus été perçues comme des simulacres de communications destinées avant tout à l'enseignant, mais comme l'occasion d'avoir de réels échanges entre pairs et de construire à plusieurs des connaissances communes. L'exemple le plus significatif est la discussion qui a eu lieu à propos de l'utilisation en classe du logiciel lexicométrique Tropes (activité $\mathrm{n}^{\circ} 11$ ). Guidés par quelques questions, les étudiants ont rapidement fait le point sur le sujet, abordant successivement les caractéristiques du logiciel (il met en lumière des aspects qui passent inaperçus à la lecture, mais ses analyses sont toutefois insuffisantes et pas toujours claires) et l'intérêt qu'il peut représenter en classe (comparaison de textes, étude d'un mot et de ses contextes, travail sur les champs sémantiques et lexicaux, aide à l'écriture, etc.) ou pour l'enseignant (aide à la conception d'exercices par exemple). Ils sont parvenus à cette occasion à tenir compte tout à la fois de la consigne (faire l'analyse d'un article de presse, la comparer à celle faite par Tropes, et donner quelques suggestions d'utilisation de ce logiciel) et à prendre en considération les remarques et propositions faites par leurs camarades. Ce dont on se rend bien compte dans le court extrait suivant (caractéristiques du logiciel et utilisation possibles en italique, dimension intertextuelle en souligné) : 


\section{Tropes pour un premier $\backslash$ "défrichagel" du texte}

[...] Nos deux analyses avaient des points communs: nous avions tous les deux détecté le type du texte (narratif) ou ll'usage fréquent $\mathrm{d}$ ''adjectifs (pour faire revivre les émotions de la veille). Mais Tropes a aussi mis en évidence des éléments auxquels je $n$ '’avais pas pensés et qui maintenant me sautent aux yeux [...] A première vue, ce programme me semble très utile pour une analyse globale du texte [...] Par contre, $j \backslash$ 'ai des doutes sur la performance de cette analyse, si on veut "creuser un peu plus profond" dans le texte $[\ldots]$.

I.

re: Tropes pour un premier $\backslash$ "défrichagel" du texte

On a toutes les deux utilisé le mot global dans notre message mais pas dans le même sens. je crois qu'on est d'accord sur le fait que Tropes propose une lecture très analytique du texte. Il n'est pas sûr que les élèves arrivent ensuite à hiérarchiser les informations du texte. Il faut les aider. Je ne pense pas que l'approche par Tropes doive se faire au début du contact avec le texte pour les élèves mais plutôt en vérification d'hypothèses [...].

C.

re: Tropes pour un premier $\backslash$ "défrichagel" du texte

tu as raison en ce qui concerne le regard critique du prof, regard que n'a pas le logiciel qui agit comme un robot qui collecte des infos et les restitue le plus rapidement possible $[\ldots]$

A.-M.

re: Tropes pour un premier \"défrichagel" du texte

C'est un gain de temps et là on est tous d'accord je crois [...]. J'avais en effet pris un texte assez long et il a défricher pas mal de choses [...] Je suis d'accord pour dire qu'il a quand même des limites, puisqu'il fait des petites erreurs d'analyse (qui n'en fait pas??) mais c'est au professeur de corriger et de garder le meilleur de cette analyse! [...] Ce logiciel serait intéressant pour faire des lacunaires [...] Comme l'a dit Lucie, c'est dommage qu'on ne l'ait pas connu avant didactique de l'écrit, on aurait gagné du temps...tant pis... Est-ce que quelqu'un l'a utilisé avec un texte un peu plus littéraire, poétique par exemple??? [...]

A.

\section{Sur l'autonomie}

Au premier abord, le jugement porté par les étudiants sur le dispositif est assez positif. Près de $80 \%$ des 28 étudiants qui ont répondu au questionnaire ${ }^{3}$ estiment que les diaporamas se sont révélés de bons supports pour comprendre les notions abordées et $64 \%$ qu'ils leur ont permis de mieux saisir les relations entre les réflexions théoriques et les applications. La possibilité de pouvoir accéder à partir de la plateforme aux supports de cours et à l'agenda a semblé très utile à $67 \%$ d'entre eux. C'est encore le même pourcentage d'étudiants qui déclarent que le nombre d'heures de tutorat informatique et pédagogique était suffisant. 24 étudiants $(85 \%)$ sur 28 considèrent que les tâches proposées entre chaque séance de cours ont été utiles, et $23(82 \%)$ qu'elles leur ont permis de saisir plus finement les points

3. Les personnes qui ont répondu au questionnaire sont les mêmes qui ont participé régulièrement aux activités proposées sur le forum. 
théoriques vus en cours. A propos des forums de discussion, $85 \%$ d'entre eux disent qu'ils ont été utilisés à bon escient dans la formation. Enfin, concernant les principes sur lesquels repose l'évaluation, 23 étudiants $(82 \%)$ estiment satisfaisant d'avoir eu à choisir 3 tâches parmi 4 proposées, 20 (71\%) d'avoir dû élaborer pour chaque scénario pédagogique un argumentaire justifiant leurs choix et leur démarche, $21(75 \%)$ d'être dispensés d'une tâche si la participation aux activités proposées sur la plateforme est régulière. Les seules réserves sont relatives à la charge de travail, à certains aspects matériels et organisationnels (absence de mise à niveau en informatique, nombre d'heures de libre accès insuffisant pour ceux qui ne possèdent pas d'ordinateur) et surtout au cours magistral. Sans que je puisse donner de chiffres précis car les remarques ont été faites en différents endroits du questionnaire, dans des espaces de questionnement ouverts, le cours magistral a semblé trop en rupture par rapport au tutorat et à la plateforme. Beaucoup auraient en effet apprécié qu'il y ait plus d'interactions en cours.

Mais tout au plus peut-on déduire de ces résultats que la majorité des étudiants s'est sentie à l'aise dans le dispositif, qu'ils ont réussi à trouver leurs marques. Mais qu'en est-il de leur façon de travailler? Leur culture d'apprentissage a-t-elle évolué au fil du semestre? Si une partie d'entre eux estiment, dans les réponses aux questions ouvertes, que l'alternance de cours magistraux, de séances de tutorat et d'échanges sur la plateforme Dokeos leur a permis de travailler de façon plus autonome (3), d'individualiser l'apprentissage (2), de travailler chez eux (1), d'avoir plus de liberté (4), ou bien encore de structurer leur travail d'une semaine sur l'autre (1), $57 \%$ disent que le dispositif n'a eu aucun impact sur leur façon de travailler et près de $68 \%$ qu'il n'a en rien modifié leur façon d'apprendre. Comment interpréter l'écart entre ces deux groupes de résultats ? Comment expliquer que les étudiants aient jugé l'effet relativement faible du dispositif sur leur comportement cognitif, même si l'on peut légitimement se poser la question de la capacité des étudiants à s'auto-évaluer dans ce domaine ? Comment expliquer enfin que dans la partie du questionnaire consacrée à la dimension professionnalisante du cours, près de $80 \%$ d'entre eux se disent malgré tout convaincus de l'intérêt d'utiliser les TICE dans l'enseignement/apprentissage du FLE?

On peut avancer que cela soit le résultat d'un certain manque de recul. Dans le souci de recueillir un maximum de questionnaires, ceux-ci ont été mis à la disposition des étudiants sur la plateforme juste avant la dernière séance de cours, alors qu'à cette époque de l'année, la plupart d'entre eux étaient absorbés dans la réalisation des différents dossiers qu'ils avaient à rendre avant la fin du semestre. $\mathrm{Nul}$ doute que les résultats auraient été différents si le sondage avait eu lieu à l'issue de la formation. Mais combien auraient pris le temps de répondre ? Le manque de recul peut aussi être imputé au modèle pédagogique choisi. Attendre de l'alternance des démarches explicites et implicites qu'elles aboutissent à une prise de conscience de la part des étudiants, non seulement de ce qu'implique pour l'enseignant l'autonomisation des apprenants de FLE, mais également de ce que cela signifie pour eux en tant qu'apprenants, n'était peut-être pas la meilleure solution. Compte 
tenu de la complexité du dispositif mis en œuvre, c'était ajouter une difficulté supplémentaire. Comme l'analyse Anne Sellier :

« La mise en relation entre les théories abordées en cours, la réflexion sur son apprentissage et sur le processus d'acquisition de l'apprenant était complexe à mettre en place [...] Il était donc très compliqué de demander aux étudiants de comprendre dans un premier temps les théories, de leur laisser le temps de les assimiler, puis de les confronter à leur expérience présente » (Sellier 2005, 30).

Le double enjeu de cette formation (former à la fois des apprenants et des (futurs) enseignants), aurait nécessité dès le départ d'informer les étudiants de la particularité de ce cours qui comprend à la fois une formation disciplinaire et une formation à l'autonomie. Pour Henri Holec, on peut vouloir faire en sorte que l'apprenant apprenne à apprendre avant ou pendant l'apprentissage disciplinaire, « et on peut choisir des procédures qui le conduisent à apprendre à apprendre de manière explicite, déductive [...] ou à apprendre à apprendre empiriquement, en « faisant », c'est-à-dire en prenant des décisions concernant un apprentissage en cours » (Holec, 1990, 84-85). C'est manifestement la première solution qu'il convient de privilégier pour cette formation, en précisant bien dès le départ les termes du contrat didactique. Dans cette perspective, il semble préférable d'opter pour un moyen permettant la réflexion tout au long du processus d'apprentissage (journal de bord assorti de la rédaction d'un rapport réflexif (Soubrié, 2006)) au lieu d'un questionnaire qui ne permet pas une distanciation suffisante.

\section{Conclusion}

Malgré le fait que l'objectif d'autonomisation des étudiants n'ait pas été atteint de façon entièrement satisfaisante, le bilan reste positif. En devenant obligatoire, le cours s'est ouvert à un public diversifié, et cela a permis aux personnes qui entretenaient jusque là des relations distantes, voire méfiantes, à l'égard des TICE, d'être au bout du compte agréablement surprises par les potentialités offertes par ces nouveaux outils en matière d'innovation pédagogique. Cela m'a également conduit à repenser le dispositif et à intégrer la problématique de l'autonomie, sans pour autant perdre de vue les apprentissages collectifs qui occupaient déjà les années passées une place importante.

En 2005/2006, ce dispositif a été reconduit avec toutefois deux aménagements importants : mise en place d'un journal de formation, que les étudiants devaient remplir à des échéances fixées à l'avance, et réduction du nombre d'heures de cours magistraux (16 h au lieu de $24 \mathrm{~h}$ ) (Soubrié, 2006). Ce qui ressort globalement de ces deux années de fonctionnement, c'est combien l' «allègement » de la formation, par la réduction du nombre d'heures de cours proprement dit, se double d'un investissement beaucoup plus lourd de la part de l'enseignant et des étudiants dans 
la formation. Dans la mesure tout du moins où l'on opte pour une pédagogie résolument active qui accorde une place centrale à l'activité de l'apprenant dans la construction des connaissances. L'hybride en effet, de mon point de vue, est moins un moyen de résoudre des problèmes logistiques qu'une façon de repenser la relation pédagogique (Charlier, 2004).

L'externalisation de la formation par le recours à une plateforme de e-learning a ainsi permis de « ralentir le temps de l'apprentissage » (Bruillard, 2003). Les tâches collectives en ligne, qui ont pris principalement la forme de débats à l'intérieur de forums de discussion, ont favorisé une participation active et un engagement constant de la part des étudiants. D'autant plus que les activités proposées à distance étaient réellement intégrées dans le présentiel, c'est-à-dire qu'il était impossible de suivre un cours sur place dès lors que les activités en ligne n'avaient pas été réalisées.

Dans ce modèle, l'encadrement joue un rôle fondamental. On l'a vu, l'autonomie ne se décrète pas. Il importe d'établir les conditions de son développement, conditions qui passent par une intense pratique réflexive, aussi bien en ce qui concerne les contenus, la manière d'apprendre que le dispositif de formation en lui-même. Les tutorats, conçus comme un accompagnement personnalisé, un peu comme dans la pédagogie du conseil (cf. CRAPEL), ont très bien répondu à cette attente. L'inconvénient est qu'ils ont été dévoreurs de temps, $\mathrm{du}$ fait que les étudiants, en raison de leur nombre, ont dû être répartis dans des groupes. Sans compter le travail de mise en ligne de contenus, d'animation et de tutorat sur la plateforme (documents, tâches, annonces, etc.). Or, s'il existe à l'université des modes de calcul qui permettent de distinguer les TP et TD des cours magistraux, aucune grille ne permet à l'heure actuelle d'évaluer le temps et l'énergie consacrés au suivi d'un dispositif de formation ouverte et partiellement à distance.

C'est pourquoi l'on peut se poser la question de la reproductibilité du modèle et surtout de sa généralisation. Tant, en effet, que ce type d'initiative reste minoritaire, que les conditions matérielles le permettent (salles informatiques en libre-accès par exemple) et que l'enseignant en charge du dispositif y trouve un intérêt personnel (comme c'est mon cas par exemple, dans la mesure où ces expérimentations nourrissent mon travail de recherche), rien ne s'oppose à l'élaboration de configurations de ce type. L'institutionnalisation de telles pratiques poserait en revanche de redoutables difficultés en termes d'équipement, d'organisation, d'ingénierie, de calcul du service enseignant etc. Mais le jeu n'en vaut-il pas la chandelle?

Comme cela a été souligné lors du colloque JOCAIR qui s'est tenu à Amiens les 6 et 7 juillet 2006, les TICE permettent pour la première fois d'introduire à l'université des problématiques d'ordre pédagogique. Il est certain que l' «enrichissement» du présentiel rompt de manière assez radicale avec l'enseignement tel qu'il est encore conçu dans l'enseignement supérieur. Les bénéfices de la mise en place de tels dispositifs de formation qui ont pu être observés à travers diverses expériences, qui vont tous dans le sens d'une 
amélioration de la qualité de la prestation pédagogique, ne pourront à l'avenir qu'encourager les responsables de formation à étudier sérieusement ces nouvelles modalités d'enseignement/apprentissage.

\section{Bibliographie}

Albero B., «L'autoformation dans les dispositifs de formation ouverte et à distance : instrumenter le développement de l'autonomie dans les apprentissages », Saleh I., Lepage D., Bouyadi S., Les TIC au cour de l'enseignement supérieur, Actes de la journée d'étude du 12 novembre 2002, Laboratoire Paragraphe, Université Paris VIII, VincennesSaint Denis, coll. Actes Huit, 2003, p. 139-159,

http://archivetematice.ccsd.cnrs.fr/docs/00/00/17/75/PDF/AlberoVincennes.pdf

Albero B., "L'autoformation en contexte institutionnel : entre la contingence et l'utopie », G. Le Meur, Université Ouverte, Formation virtuelle et apprentissage, Communications francophones du $5^{e}$ colloque européen sur l'autoformation à Barcelone, 16-18 décembre 1999, Paris, L'Harmattan, 2002, p. 459-483, http://archivetematice.ccsd.cnrs.fr/docs/00/00/17/74/PDF/AlberoBarcelone.pdf

Bruillard E., «Lorsque la distance favorise le temps et la réflexion », Medialog, mai 2003, n 46, CRDP, Académie de Créteil, 2003, p. 53-54.

Charlier, B., Deschryver, N., Peraya, D., « Articuler présence et distance, une autre manière de penser l'apprentissage universitaire », Actes du colloque de l'AIPU, Marrakech, avril 2004, http://www.unifr.ch/didactic/article.php3?id_article=13.

Daele A., Denis B., Deschryver N., Lusalusa S., Quintana J., Serra N., Willem C., « Quels apprentissages pour de futurs enseignants dans le cadre d'une communauté d'apprentissage virtuelle?», 25 conférence annuelle ATEE, Barcelone, août 2000, http://www.det.fundp.ac.be/ ada/docs/2000atee.doc

Degache C., « De quelques enjeux de l'apprentissage en autonomie en contexte universitaire : pistes de réflexion et propositions », H. Greven-Borde, D. Spalding-Andréolle, L'apprentissage des langues en autonomie, Choix, approches, questionnements, CERELC - PPF langues et cultures, Université Stendhal, Grenoble, 2003, p. 173-198.

Holec H., «Qu'est-ce qu'apprendre à apprendre?», Mélanges pédagogiques, CRAPEL/Université de Nancy, 1990.

Jeanneret Y., «Les politiques de l'invisible. Du mythe de l'intégration à la fabrique de l'évidence ", Sylvie Leleu-Merviel, Document numérique, Nouvelles écritures, vol. 5, $\mathrm{n}^{\circ}$ 1-2, Hermès, Paris, 2002, p. 155-180.

Jeanneret Y., Y a-t-il (vraiment) des technologies de l'information?, Presses du Septentrion, 2000.

Lameul G., «Ingénierie de Formation Continue de l'IUFM de BRETAGNE : questionnement relatif à la notion de dispositif $»$, Actes $d u \sigma^{e}$ colloque sur l'autoformation, Montpellier, 3-5 décembre 2001, http://www.educagri.fr/reseaux/cdr/colloq2001/Lameul.pdf

Legros D., Crinon J., Psychologie des apprentissages et multmédia, Colin, Paris, 2002. 
Linard M., «Autoformation, éthique et technologies : enjeux et paradoxes de l'autonomie », Albero B., Autoformation et enseignement supérieur, Hermès/Lavoisier, p. 241-263, http://www.epathie.com/Linard\%202003.pdf

Linard M., "Conception de dispositifs et changement de paradigme en formation », Education Permanente, $\mathrm{n}^{\circ} 152$, octobre, « Regards multiples sur les nouveaux dispositifs de formation », 2002, p. 143-145, www.epathie.com/Linard\%202002.pdf

Mangenot F., «Tâches et coopération dans deux dispositifs universitaire de formation à distance », Alsic, vol. 6, n 1 , Juin 2003, p. 109-125, http://alsic.u-strasbg.fr/Num10/mangenot/alsic_n10-rec10.htm

Ollivier B., Internet, multimédia : ça change quoi dans la réalité ? INRP, Paris, 2000.

Portine H., «L'autonomie de l'apprenant en question », Alsic, vol. 1, n 1, juin 1998, http://alsic.u-strasbg.fr/Num1/portine/alsic_n01-poil.htm

Salembier P., «Cognition(s) : Situées, Distribuée, Socialement Partagée,etc.», Bulletin duLCPE, 1, Ecole Normale Supérieure, Paris.

Sellier A., Les représentations sur les TICE des étudiants de mastère FLE, Mémoire de première année de master de sciences du langage, spécialité FLE, Université StendhalGrenoble 3, mai 2005.

Souchier E., Jeanneret Y., Le Marec J., Lire, écrire, récrire. Objets, signes et pratiques des médias informatisés, Bibliothèque publique d'information/Centre Pompidou, Paris, 2003.

Soubrié T., «AUKAPIWeb, auxiliaire pour l'appropriation de l'information sur le Web : du traitement automatique de l'information à sa manipulation », Polity Y., Henneron G., Palermiti R., L'organisation des connaissances, approches conceptuelles, Actes du congrès Isko-France qui s'est tenu à Grenoble les 3 et 4 juillet 2003, L'Harmattan, 2005, p. 253-256.

Soubrié T., «Dispositif de formation ouverte à l'utilisation des TICE dans l'enseignement/apprentissage du FLE en master», $7^{\mathrm{e}}$ colloque européen sur l'autoformation, 18-20 mai 2006, Toulouse, ENFA, http://www.enfa.fr/autoformation/rub-comm/pdf/soubrie.pdf 